\title{
Caracterización de lineamientos estructurales y sus implicaciones hidrogeológicas en la cuenca de Villa Hidalgo (San Luis Potosí) integrando métodos geofísicos potenciales
}

Fred Y. Pérez-Corona, Héctor López-Loera, Emilia Fregoso-Becerra, Vsevolod Yutsis, Víctor Julián Martínez-Ruíz, Pablo Dávila-Harris

Fred Y. Pérez-Corona

fred.perez@ipicyt.edu.mx

Posgrado de Geociencias Aplicadas, Instituto Potosino de Investigación Científica y Tecnológica, A.C., Camino a la Presa San José, \# 2055, Colonia Lomas 4a. Sección, G.P. 78216, San Luis Potosí, México.

\section{Héctor López-Loera \\ Vsevolod Yutsis \\ Víctor Julián Martínez-Ruíz \\ Pablo Dávila-Harris \\ División de Geociencias Aplicadas, Instituto Potosino de Investigación Científica y Tecno- lógica, A.C., Camino a la Presa San José, \# 2055, Colonia Lomas 4a. Sección, C.P. 78216 , San Luis Potosí, México.}

\section{Emilia Fregoso-Becerra}

Centro Universitario de Ciencias Exactas e Ingeniería (CUCEI), Departamento de Matemáticas de la Universidad de Guadalajara, Blvd. Marcelino García Barragán, \# 1421, Esq. Calzada Olímpica, C.P. 44430, Guadalajara, Jalisco, México

BOL. SOC. GEOL. MEX. 2017

VOL. 69 NO. 3

P. $555-576$

\section{RESUMEN}

A partir de la integración y procesamiento de conjuntos de datos potenciales (magnetometría y gravimetría), se interpretaron lineamientos geofísico-estructurales relacionados a fallas de rumbo noroeste y norte los cuales afectan la cuenca de Villa Hidalgo. La interpretación indica una tendencia NW-SE asociada a la Orogenia Laramide y a zonas de cizalla post-laramídicas.

Las direcciones N-S corresponden a fallas normales ligadas a esfuerzos extensionales originando zonas de debilidad, sirviendo como conductos para el emplazamiento de rocas volcánicas, las cuales posteriormente sufrieron subsidencia formando grábenes como el de Corcovada y Peotillos.

Los análisis piezométricos y la modelación geofísica muestran la existencia de diversos acuíferos cuyo almacenamiento y circulación se da a través de material aluvial del Cuaternario, fallas y/o fracturas en rocas volcánicas del Oligoceno y/o rocas sedimentarias del Cretácico (Albiano-Cenomaniano y Maastrichtiano-Campaniano), presentando una dirección preferencial de flujo Sur-Norte.

La porción noreste de la cuenca se caracteriza por tener los valores más bajos de elevación topográfica, así como los valores más bajos de densidad y magnetización de las diferentes unidades geológicas presentes en el subsuelo, vinculados a la principal zona de descarga, en donde el cruce de los sistemas de fallas NW-SE y N-S han provocado una depresión conectando al sistema acuífero de Villa Hidalgo con el sistema acuífero Cerritos.

La correlación entre los datos geofísicos, los diferentes sistemas de falla y los lineamientos geofísico-estructurales sugieren que la Guenca de Villa Hidalgo es una posible zona transtensional, la cual controla el funcionamiento hidrogeológico del sistema acuífero.

Palabras clave: Guenca de Villa Hidalgo, métodos potenciales, lineamientos geofísico-estructurales, funcionamiento hidrogeológico.

\section{ABSTRACT}

Based on the integration and processing of potential datasets (Magnetic and Gravity), geophysical-structural lineaments were interpreted to be related to faults that strike northwest and north affecting the Villa Hidalgo basin. The interpretation indicates that $\mathcal{N W}$-SE trend is associated with Laramide Orogeny and post-laramid shear zones. The $\mathcal{N}-S$ directions correspond to normal faults linked to extensional stresses that gave rise to zones of weakness serving as conduits for the placement of volcanic rocks, which later collapsed forming grabens such as Corcovada and Peotillos. Piezometric analyzes and geophysical modeling show the existence of several aquifers whose storage and circulation occurs through alluvial material of the Quaternary, faults and fractures in volcanic rocks of the Oligocene and sedimentary rocks of the Cretaceous (Albian-Cenomanian and Maastrichtian-Campanian), presenting a preferred direction of South-North flow.

The northeast portion of the basin is characterized by having the lowest values of topographical elevation, as well as lowest density and magnetization values of the different geological units present in the subsoil, linked to the main discharge zone, where the crossing of the $\mathcal{N} W$-SE and $\mathcal{N}-S$ fault systems has caused the connection of the Aquifer system of Villa Hidalgo with the Cerritos aquifer system.

The correlation between the geophysical data, the different fault systems and the geophysical-structural lineaments suggest that the Villa Hidalgo basin is a possible transtensional zone that controls the hydrogeological functioning of the aquifer system.

Keywords: Villa Hidalgo basin, potential methods, Geophysical-structural lineaments, hydrogeological functioning. 


\section{Introducción}

Gran parte de los sistemas acuíferos del centro de México se localizan en valles tectónicos, v.g. el Acuífero de San Luis Potosí y el Acuífero de Villa de Reyes (Ramos-Leal et al., 2007), tal es el caso de la Cuenca de Villa Hidalgo $(\mathrm{CVH})$, localizándose 30 kilómetros al norte de la ciudad capital de San Luis Potosí y tiene una área de 1490 km² (Figura 1). Este tipo de escenarios geológicos son dominados por sistemas de fallas que generalmente originan fosas y pilares tectónicos controlando la dinámica del agua subterránea, pero debido a que la mayoría de estas estructuras se encuentran sepultadas por material de relleno, no es posible identificar superficialmente la geometría y funcionamiento de los sistemas acuíferos, provocando que la localización de fuentes de abastecimiento de agua no sea exitosa (Ramos-Leal et al., 2007).

Ante esta problemática se hace necesaria la utilización de técnicas indirectas de exploración como los métodos geofísicos potenciales (gravimetría y magnetometría). Diversos autores han realizado importantes trabajos de cartografía en la zona de estudio (e.g. Aranda-Gómez y Labarthe-Hernández, 1977; Zapata Zapata y Pérez-Venzor, 1979; Barboza-Gudiño et al., 2002; López-Doncel et al., 2007; Tristán-González et al., 2008), identificando lineamientos locales y regionales utilizando imágenes satelitales y Modelos Digitales de Elevación (MDE), los cuales permitieron inferir rastros de zonas de falla debido a la pobre o nula exposición de rocas del basamento en el valle de la CVH. Sin embargo, utilizando gravimetría y magnetometría es posible analizar y detallar las zonas de falla reportadas, determinando los contrastes de las propiedades físicas existentes entre los contactos de las diferentes rocas o formaciones geológicas del subsuelo (Reynolds et al., 1990; Blakely, 1995)

En este trabajo además se identificó la geometría del sistema acuífero y el control estructural del funcionamiento hidrogeológico en la zona de estudio, así como su posible interconexión con otras cuencas.

\section{Marco Geológico}

La CVH se sitúa dentro de la zona límite de las provincias de la Mesa Central (MC) en el oeste, conformando a su vez la parte sur de la provincia extensional Basin and Range (Aranda-Gómez et al., 2000; Nieto-Samaniego et al., 2005; Tristán-González et al., 2009).

La MC presenta un basamento de secuencias marinas del Triásico de la Formación Zacatecas constituida por una alternancia rítmica (turbidítica) de areniscas de grano medio a fino, lutitas y ocasionalmente conglomerática de color gris oscuro (Martínez-Pérez, 1972; Zavala-Monsiváis, et al., 2012), correlacionándose con la Formación La Ballena (Silva-Romo, 1994) y la Formación Taray (Córdoba- Méndez, 1964), las cuales se asocian a un sistema de abanico submarino del margen paleo-pacífico de Norte América (Silva-Romo et al., 2000; Hoppe et al., 2002; Centeno-García, 2005; Barboza-Gudiño et al., 2010) denominado Abanico Potosí.

En la localidad de Real de Catorce se ha encontrado que el basamento está constituido por la Formación Zacatecas correspondientes al Triásico Superior (Barboza-Gudiño et al., 2010), por lo que se infiere que ésta sucesión de sedimento puede presentarse a profundidad en la $\mathrm{CVH}$.

$\mathrm{Al}$ este de la zona límite del área de estudio se encuentra el cinturón plegado de la Sierra Madre Oriental (SMOR) (Figura 1a), constituida durante el Triásico por sedimentos de origen continental correspondientes a la Formación Huizachal (Imlay et al., 1948) o a la Formación La Boca (Mixon et al., 1995) compuestas por una sucesión de capas rojas depositadas en un régimen extensional durante y después de actividad de arco magmático (Barboza-Gudiño et al., 2008; Barboza-Gudiño et al., 2010).

La sucesión triásica de la MC y de la SMOR sobreyace a un basamento granulítico Grenvilliano, caracterizado a partir de diferentes estudios de xenolitos contenidos en rocas volcánicas del Cenozoico ubicadas al oeste de San Luis Potosí (Schaaf 
et al., 1994; Barboza-Gudiño et al., 2010).

La CVH también se encuentra inmersa en el margen de los elementos paleo-geográficos del Cretácico de la Plataforma Valles-San Luis Potosí (PVSLP) al oriente y la Cuenca Mesozoica del Centro de México (CMCM) al poniente (Figura
$1 b)$.

Los grandes espesores de los depósitos de calizas arrecifales y post-arrecifales que conforman la PVSLP evidencian la ocurrencia de movimientos de subsidencia pausada pero constante del bloque de la plataforma y un relativo ascenso lento del

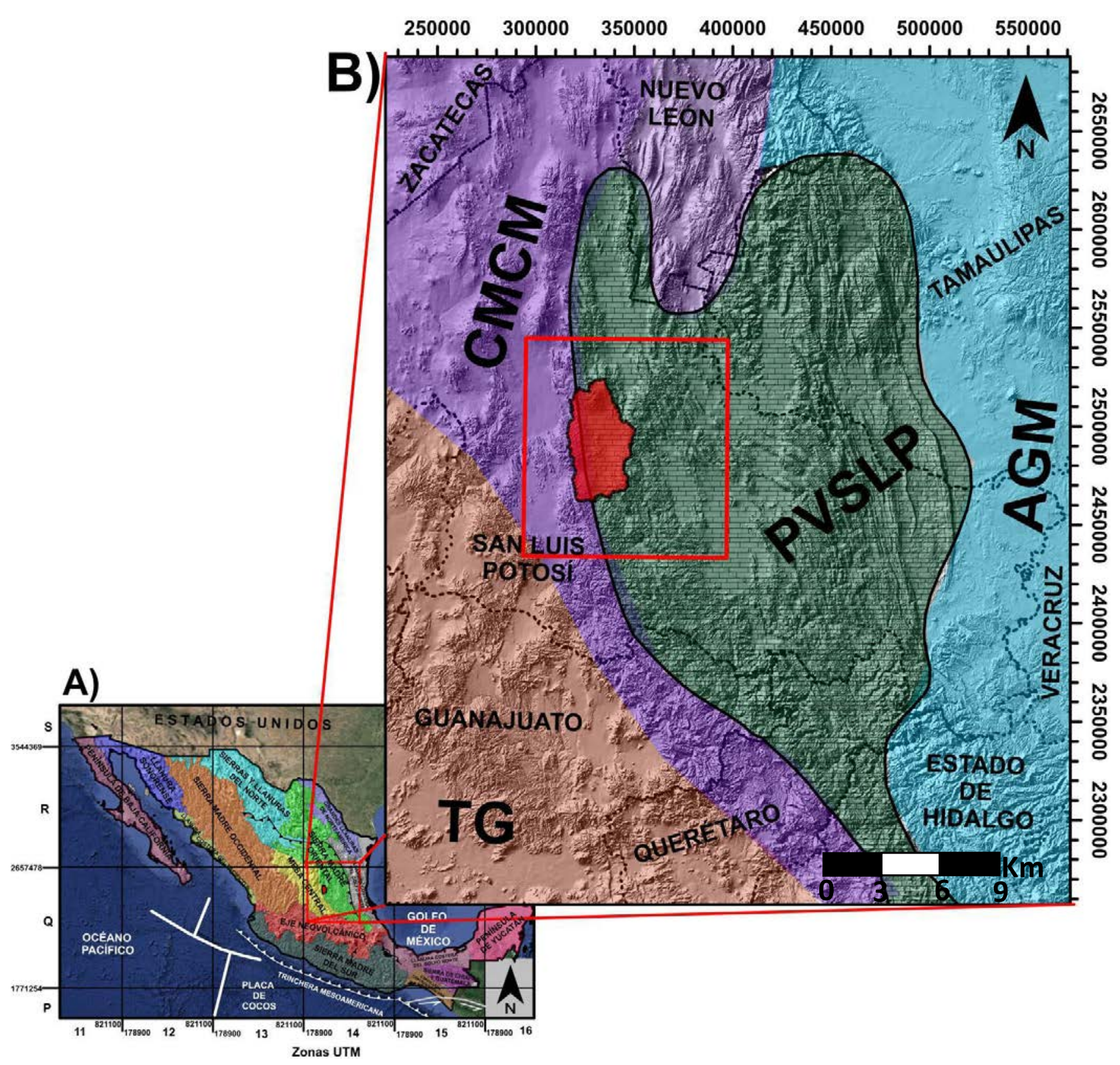

Figura 1 Mapa de ubicación del área de estudio. A) Localización entre las provincias de la Sierra Madre Oriental y La Mesa Central, B) Localización de los elementos paleogeográficos de la Plataforma Valles-San Luis Potosí (PVLSP) y la Cuenca Mesozoica del Centro de México (CMCM), también se muestran los límites del Terreno Guerrero (TG) y el Antiguo Golfo de México (AMG).. 
nivel del mar (Barboza-Gudiño et al., 2002).

La geología local del área (Figura 2) está representada por: rocas sedimentarias marinas carbonatadas de composición calcárea, arrecifales de la Formación El Abra (Albiano-Cenomaniano), calcáreas-arcillosas con pedernal de la Formación Cuesta del Cura (Albiano-Cenomaniano), arcillo-calcáreas de la Formación Indidura (Turoniano-Santoniano), arcillo-calcáreas carbonosas de la Formación Soyatal (Turoniano-Santoniano), lutitas y areniscas de la Formación Cárdenas
(Maastrichtiano-Campaniano).

Estas rocas sedimentarias marinas, se encuentran plegadas, con ejes de rumbo NW-SE, recostados hacia el NE, formando las sierras de Peotillos, El Coro, La Tapona, El Peyote y Álvarez (Barboza-Gudiño et al., 2002; Tristán-González et al., 2008).

Sobreyaciendo discordantemente a las rocas sedimentarias del Cretácico existe una secuencia volcánica constituida por lavas formando domos asociados a flujos piroclásticos, derrames de com-

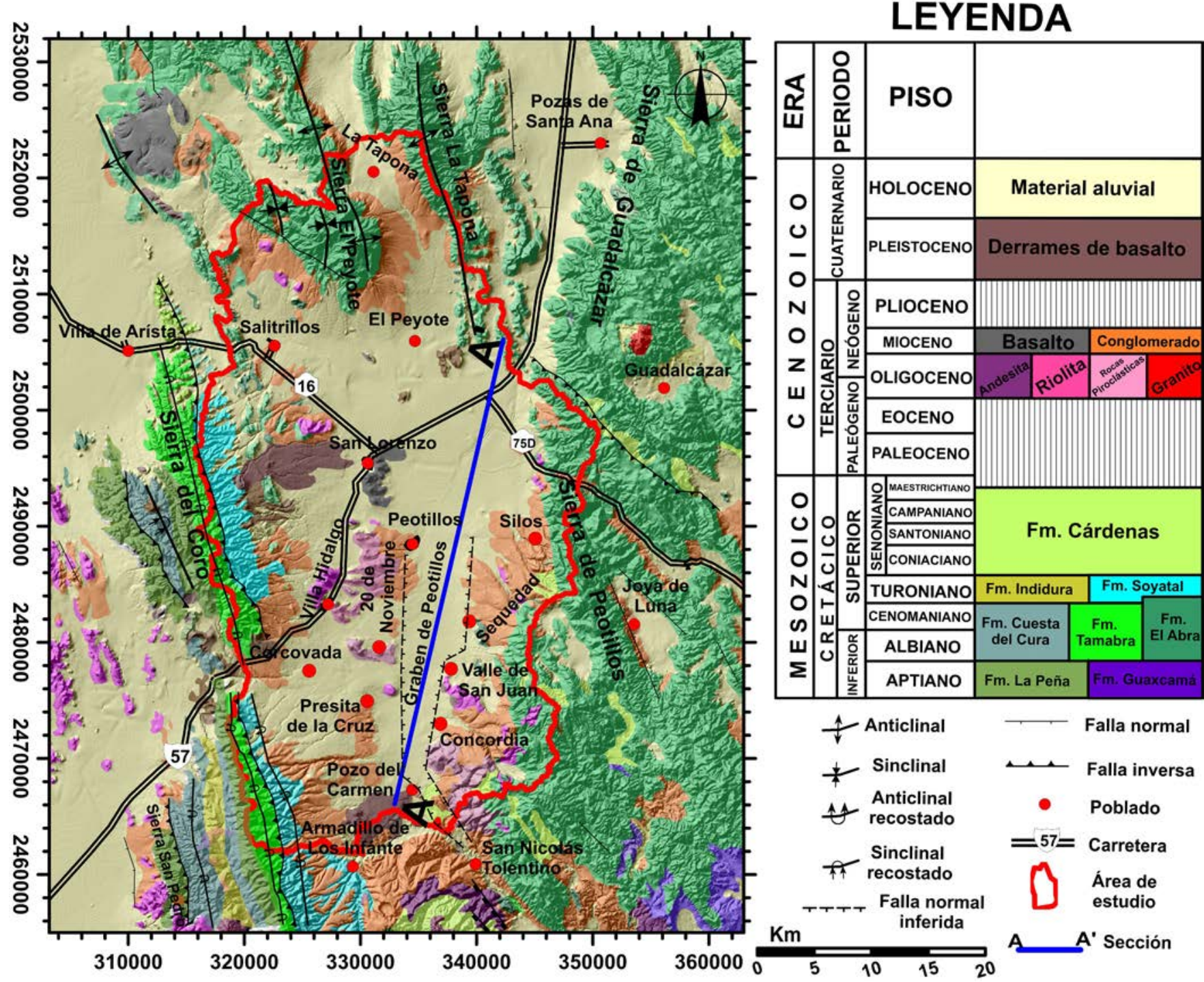

Figura 2 Plano geológico simplificado de la Cuenca de Villa Hidalgo. Modificado de la base geológica de Labarthe-Hernández et al. (1982). Obsérvese la ubicación de la sección Geofísica-Geológica A-A’. 
posición andesítica-dacítica, andesitas-basaltos e ignimbritas riolíticas del Eoceno-Oligoceno (Barboza-Gudiño et al., 2002; Tristán-González et al., 2008; Tristán-González et al., 2009).

Posteriormente en el Plioceno-Pleistoceno hubo un segundo periodo de vulcanismo basáltico originando conos cineríticos, derrames y volcanes de explosión o tipo maars. Por último se depositaron sedimentos aluviales del Cuaternario. (Aranda-Gómez, 1977; Barboza-Gudiño et al., 2002; Tristán-González et al., 2009).

\section{Exploración Geofísica}

\subsection{MAGNETOMETRÍA TERRESTRE Y AÉREA}

Los datos de magnetometría terrestre se obtuvieron del levantamiento en campo de 11 líneas medidas en superficie distribuidas a lo largo y ancho de la zona de estudio, obteniendo una longitud total de $213 \mathrm{~km}$, con 404 estaciones de medición separadas en promedio cada $500 \mathrm{~m}$ (Figura 3A), se les aplicó corrección diurna y horaria. Los datos de magnetometría aérea utilizados fueron obtenidos del Servicio Geológico Mexicano (Figura 3G). En ambos casos se les restó el IGRF (NOAA 2010) a los datos del Campo Magnético Total (CMT) obteniendo la Anomalía Magnética (AM).

Posteriormente se aplicó un filtro polinomial (FP) de segundo grado (Coons et al., 1967; Beiki et al., 2010) para obtener el Campo Magnético Residual (GMR), lo anterior se realizó bajo el criterio de que los FPs de orden bajo tienen buen ajuste con las anomalías de componentes regionales y minimizan los efectos de las anomalías residuales relacionadas a características geológicas poco profundas (Hinze et al., 2013).

El campo magnético de la tierra (inclinación y declinación) varía en función de la posición geográfica desplazando las anomalías magnetices de la fuente que las produce.

El algoritmo de Reducción al Polo (RP) (Baranov y Naudy, 1964) ubica las anomalías magnéticas directamente sobre las fuentes que las causan al simular la ubicación del área de estudio en el Polo, donde la inclinación magnética es $90^{\circ}$ y la declinación es $0^{\circ}$. Cuando las áreas de estudio son pequeñas, los errores de RP asociados al uso de una sola inclinación y declinación son mínimos porque la dirección del campo magnético de la Tierra varía muy poco. Sin embargo, para estudios geológicos regionales $\left(>10000 \mathrm{~km}^{2}\right)$, estos errores pueden ser significativos (Lu et al., 2003; Hinze et al., 2013). Debido a que el área de estudio presenta un tamaño menor a $10000 \mathrm{~km}^{2}\left(1490 \mathrm{~km}^{2}\right)$ se aplicó el algoritmo de RP (Figuras 4A y 4C).

\subsection{GRAVIMETRÍA TERRESTRE Y SATELITAL}

Los datos gravimétricos terrestres se midieron en los mismos puntos de la magnetometría terrestre (Figura 3B), fueron corregidos por deriva, latitud, marea y topografía. La Anomalía de Bouguer (AB) se calculó usando una reducción de densidad de $2.67 \mathrm{~g} / \mathrm{cm}^{3}$. La $\mathrm{AB}$ de los datos de gravimetría satelital (Figura 3D) se obtuvo a través del Bureau Gravimétrique International (BGI), la cual se obtiene a partir de valores satelitales y del modelo gravitacional terrestre EGM2008 elaborado por la NGA (National Geospatial-Intelligence Agency) (Pavlis et al., 2012). Dichos datos fueron corregidos usando el código FA2BOUG desarrollado por Fullea et al. (2008).

La corrección topográfica se aplicó a una distancia de $167 \mathrm{~km}$ considerando el Modelo Digital de Elevación (MDE) ETOPO1, que tiene una resolución de 1 arco-minuto por 1 arco-minuto. A los dos conjuntos de datos se les realizó la corrección por terreno utilizando el método de Hammer (Burger, 1992), con la que se obtuvo la Anomalía Completa de Bouguer (ACB).

Para estimar la anomalía residual se aplicó un FP de segundo grado (orden bajo), que como ya se mencionó ajusta bien para efectos regionales, el cual se le sustrajo a la ACB (i.e., Reynolds, 1997) (Figura 3 B y D). 

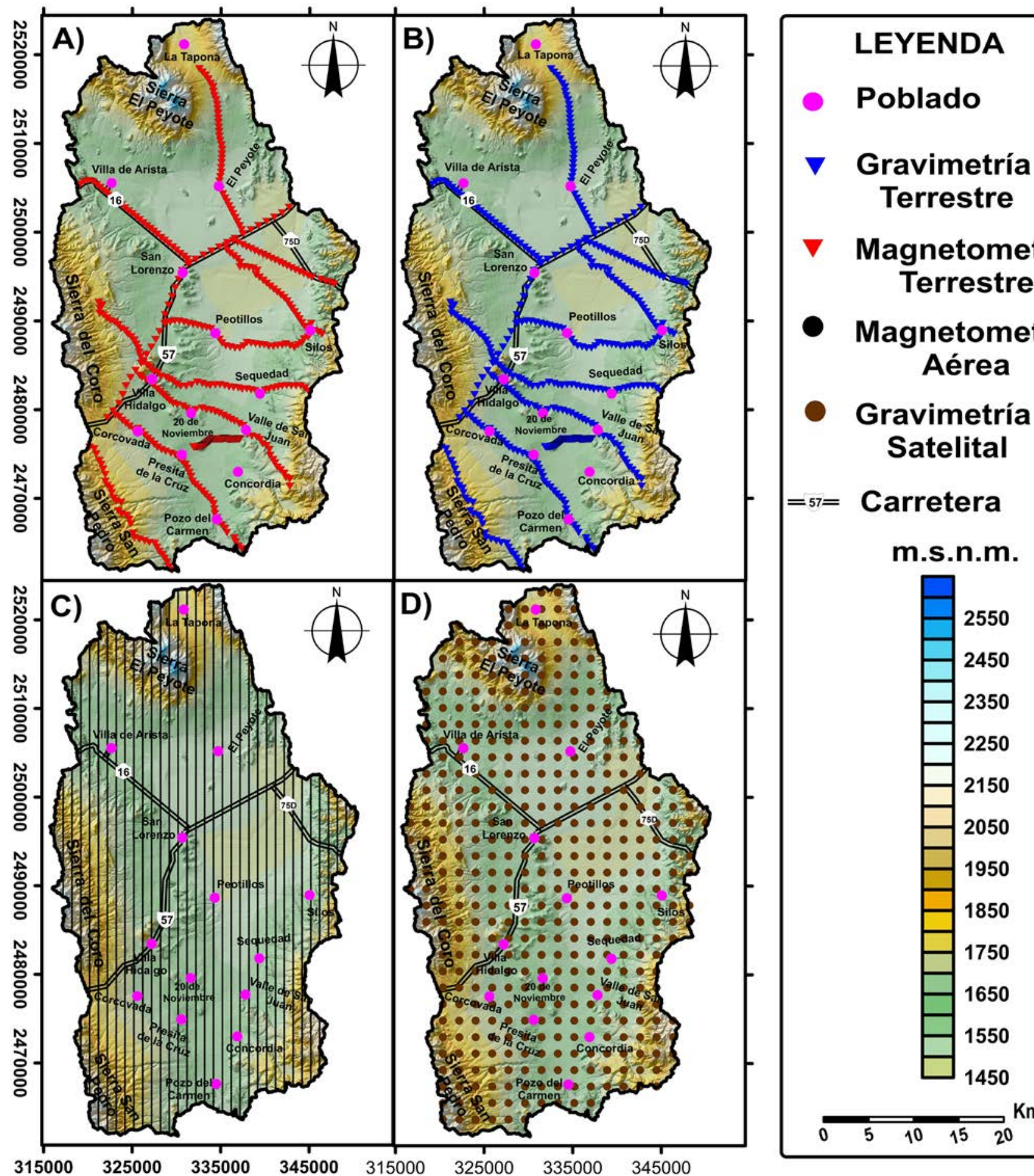

$\nabla$ Magnetometría Terrestre

Magnetometría Aérea

- Gravimetría Satelital

$=57=$ Carretera

m.s.n.m.

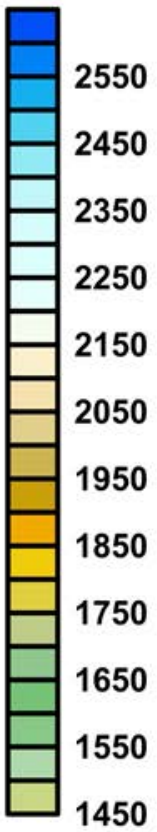

550

450

350

250

150

050

1950

850

1750

1650

550

1450

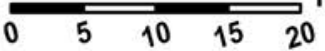

Figura 3 Distribución espacial de los diferentes conjuntos de datos. A) Magnetometría Terrestre, B) Gravimetría Terrestre, C) Magnetometría Aérea y D) Gravimetría Satelital. 


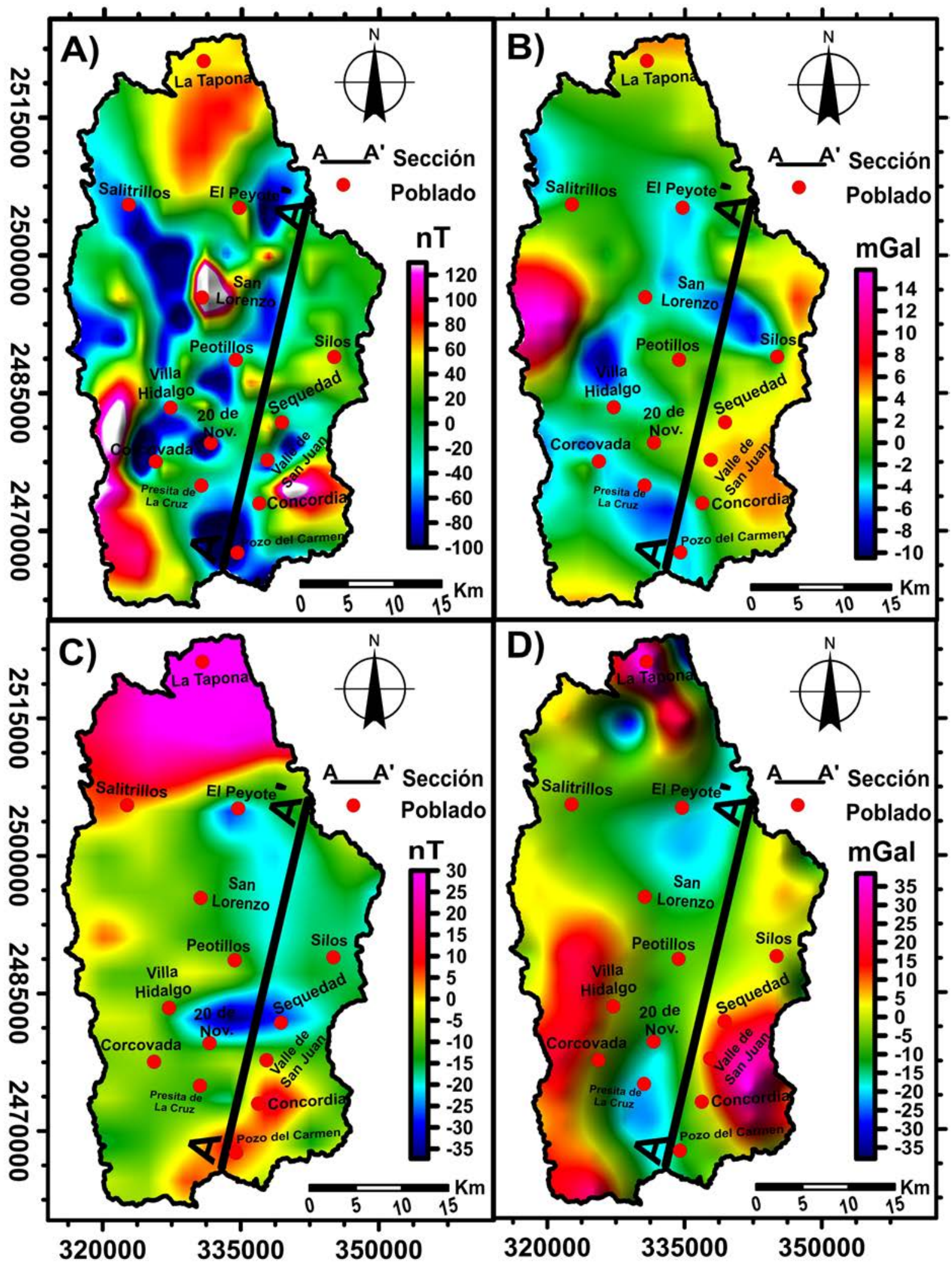

Figura 4 Planos de los métodos potenciales A) Mapa del Campo Magnético Residual Reducido al Polo de la Magnetometría Terrestre, B) Mapa que muestra la Anomalía Residual de Bouguer de la Gravimetría Terrestre, C) Campo Magnético Residual Reducido al Polo de la Magnetometría Aérea, D) Anomalía Residual de Bouguer de la Gravimetría Satelital. 


\subsection{ESPEGTRO DE POTENGIAS}

El análisis espectral de datos potenciales es una herramienta confiable para estimar profundidades medias de diferentes horizontes de los datos que tienen un cambio en las propiedades físicas (Spector y Grant, 1970; Niadu, 1970; Reddi et al., 1988). La estimación de diferentes fuentes se calcula con dicho método, tanto para datos magnéticos como para datos gravimétricos. Dos de sus principales ventajas son: 1) elimina errores causados por líneas de levantamiento que no se encuentren orientadas perpendicularmente al rumbo y 2) no depende de algún operador que el usuario tenga que seleccionar para una ventana determinada.

Además se pueden generar matrices (grids) de salida para posteriormente configurar y procesar mapas y/o imágenes que mejoran el detalle del subsuelo proporcionando información estructural, la cual de otra forma no podría ser tan evidente (Bhattacharyya, 1966; Mishra y Niadu, 1974).

En general todos los espectros de las anomalías magnéticas y gravimétricas incluyen 2 partes, una en el extremo de las bajas frecuencias, que en la mayoría de los casos es más fácil aproximar con una línea recta y denotar discontinuidades más profundas (discontinuidades de Moho y/o Conrad) y la segunda en el extremo de las altas frecuencias de carácter ondulante, denotando fuentes más superficiales (basamento y/o intrusiones). Las configuraciones para los grids del análisis del espectro de potencias muestran diferentes horizontes detectados del subsuelo para cada conjunto de datos (Figuras 5B-5E) y han sido referidas al nivel del mar.

\subsection{DEGONVOLUGIÓN DE EULER}

El método de Deconvolución de Euler (DE) fue creado por Thompson (1982) y lo aplicó en datos magnéticos reales a lo largo de diferentes perfiles. Se ha utilizado para calcular de manera automática la ubicación y profundidad de las fuentes responsables de las diferentes anomalías de campos potenciales (Bournas et al., 2003; Shepherd et al., 2006; Chennouf et al., 2007). Reid et al. (1990) desarrollaron un método equivalente para un mallado de datos (grid) y obtuvieron la ecuación 3-D de Euler:

$$
\left(x-x_{0}\right) \frac{\partial M}{\partial x}+\left(y-y_{0}\right) \frac{\partial M}{\partial y}+\left(z-z_{0}\right) \frac{\partial M}{\partial z}=-N M
$$

donde $x_{02} y_{0} \mathrm{y} z_{0}$ es la posición de una fuente, cuyo campo total $\mathrm{M}$ es detectado por $x, y, z$, y $\mathcal{N}$ es el Índice Estructural (IE), que está en función de la geometría de los cuerpos fuente que originan la anomalía.

Las derivadas horizontales $\frac{\partial M}{\partial x}, \frac{\partial M}{\partial y}$ y la derivada vertical $\frac{\partial M}{\partial Z}$ son utilizadas para aplicar el método de Euler. Considerando tres o más observaciones vecinas a la vez (una operación de ventana), se puede calcular la posición y profundidad de la fuente. Moviendo la ventana de operaciones sobre la anomalía de un lugar a otro se pueden obtener múltiples soluciones para la misma fuente.

La ventaja de la DE es la de no requerir un modelo geológico previo, sin embargo el IE es el principal parámetro de entrada, el cual implica el conocimiento a priori sobre la geometría de los cuerpos geológicos que generan las anomalías. La elección del IE determina en gran medida las profundidades calculadas. De acuerdo a diversos autores (Reid et al., 1990; Reid et al., 2003; FitzGerald et al., 2004; Hinze et al., 2013) la relación entre geometría de las fuentes e IE se pueden ver en la Tabla 1. Debido a que la geología del área presenta fallas, cabalgamientos y estructuras de buzamiento variable, el $\mathrm{IE}=0$ para datos gravimétricos e $\mathrm{IE}=1$ en datos magnéticos resultan ser los más apropiados de acuerdo con la Tabla 1. Las soluciones de Euler se han calculado utilizando los mapas de la anomalía residual de Bouguer de gravimetría terrestre y satelital, así como el CMR de magnetometría terrestre y aérea. Las ventanas utilizadas para los conjuntos de datos fueron de, $10 \times 10$ puntos ( 1 x $1 \mathrm{~km}$ ) y una tolerancia de 20 (error en $\%$ ). En la Tabla 2 se muestra un resumen de las 


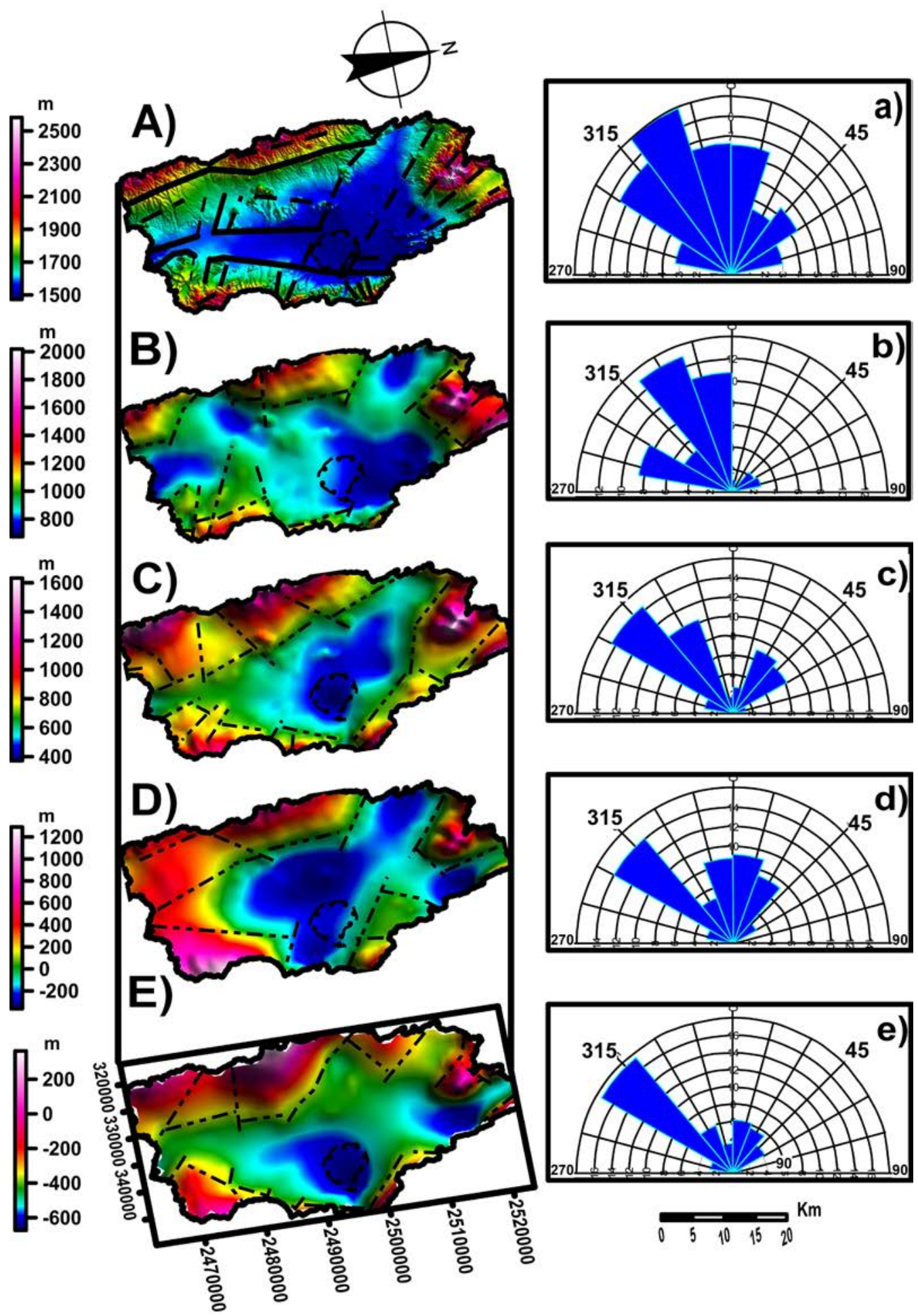

Figura 5 Planos que muestran las variaciones de la elevación en el subsuelo calculadas con el espectro de potencia junto con la tendencia de los lineamientos para cada conjunto de datos; A) Modelo Digital de Elevación, a) Lineamientos dominantes NW-SE y N-S. B) Mapa de relieve obtenido de la Gravimetría Terrestre, b) Lineamientos generales NW-SE y N-S. C) Relieve obtenidos de la Magnetometría Terrestre, c) Lineamientos en dirección NW-SE y en menor medida los NE-SW. D) Mapa de relieve de la Magnetometría Aérea, d) Lineamientos dominantes NW-SE, N-S NE-SW. E) Relieve obtenido de la Gravimetría Satelital, e) Lineamientos en dirección NW-SE y de menor frecuencia lo rumbos NE-SW. 
características y profundidades encontradas para cada conjunto datos.

En todos los casos, la mayoría de las soluciones coinciden o están agrupadas en las inmediaciones de los lineamientos gravimétricos y magnéticos de la zona de estudio (Figura 6).

\section{5. ÁNGULO DE INGLINAGIÓN (TILT ANGLE)}

El ángulo de inclinación (AI) iguala las amplitudes de la señal y los picos de los datos transformados se sitúan sobre el centro del cuerpo que los causa, es usada a menudo para detectar los bordes en contactos geológicos que pueden representar fallas (Miller y Singh, 1994). El AI es una herramienta muy útil para mapear tanto fuentes someras como fuentes profundas, se define por el arco tangente de la división de la Primera Derivada en la Vertical (PDV) entre la Derivada Horizontal Total (DHT):

$$
\mathrm{AI}=\operatorname{ARCTAN}\left(\frac{P D V}{D H T}\right)
$$

Tabla 1. La relación entre geometría de las fuentes anómalas e IE.

\begin{tabular}{|c|c|c|}
\hline $\begin{array}{c}\text { Índice } \\
\text { Estructural } \\
\text { (IE) }\end{array}$ & $\begin{array}{c}\text { FUENTE (Campo } \\
\text { Gravitatorio) }\end{array}$ & $\begin{array}{c}\text { FUENTE (Campo } \\
\text { magnético) }\end{array}$ \\
\hline 0 & Escalón/dique/falla & Contactos \\
\hline 1 & Estructuras Cilíndricas & Falla/dique/escalón \\
\hline 2 & Esferas & Estructuras Cilíndricas \\
\hline 3 & No se usa & Esferas \\
\hline
\end{tabular}

Donde DHT es el vector resultante de la combinación de las primeras derivadas horizontales en las direcciones $x, y$, acentuando los cambios laterales abruptos de las propiedades físicas de las estructuras presentes en el subsuelo (Cordell y Grauch, 1985; Verduzco, 2004).

La principal ventaja del filtro AI es que el valor de contorno igual a cero está cercano o se localiza sobre fallas, contactos o lineamientos. En el área de estudio se aplicó este filtro a los datos de magnetometría y gravimetría terrestre, así como a los datos de magnetometría aérea y gravimetría satelital, pudiéndose caracterizar y delimitar diferentes lineamientos que pueden estar asociados a fracturas, fallas y/o contactos geológicos.

\section{Resultados y Discusión}

\subsection{GARACTERIZAGIÓN DEL RELIEVE DEL SUBSUELO}

Para caracterizar el relieve del subsuelo se utilizaron las configuraciones de los grids obtenidos del espectro de potencias.

Los valores topográficos en la configuración de la gravimetría terrestre oscilan de 673 a 2018 (msnm) (Figura 5B). Los valores bajos se asocian a zonas con el mayor espesor de sedimentos de relleno correspondientes a depocentros de origen tectónico. Dichas estructuras son circundadas por montañas de origen laramídico como la Sierra El Peyote al norte (plataforma), Peotillos al este (plataforma), El Coro al oeste (cuenca) y Álvarez al SSW (cuen-

Tabla 2. Profundidad máxima y mínima de las soluciones encontradas para los diferentes índices estructurales (IE), tolerancia y tamaño de ventana $(W)$ empleadas en los diferentes conjuntos de datos.

\begin{tabular}{|c|c|c|c|c|c|}
\hline DATOS & IE & $\begin{array}{c}\text { VENTANA } \\
(\mathbf{w})\end{array}$ & $\begin{array}{c}\text { PROF. MÁXIMA } \\
(\mathbf{m s n m})\end{array}$ & $\begin{array}{c}\text { PROF. MíNIMA } \\
(\mathbf{m s n m})\end{array}$ & $\begin{array}{c}\text { TOLERANCIA } \\
\mathbf{( \% )}\end{array}$ \\
\hline Gravimetría Terrestre & 0 & 10 & 679 & 2400 & 20 \\
\hline Magnetometría Terrestre & 1 & 10 & -443 & 1747 & 20 \\
\hline Magnetometría Aérea & 1 & 10 & -580 & 1832 & 20 \\
\hline Gravimetría Satelital & 0 & 10 & -973 & 1523 & 20 \\
\hline
\end{tabular}




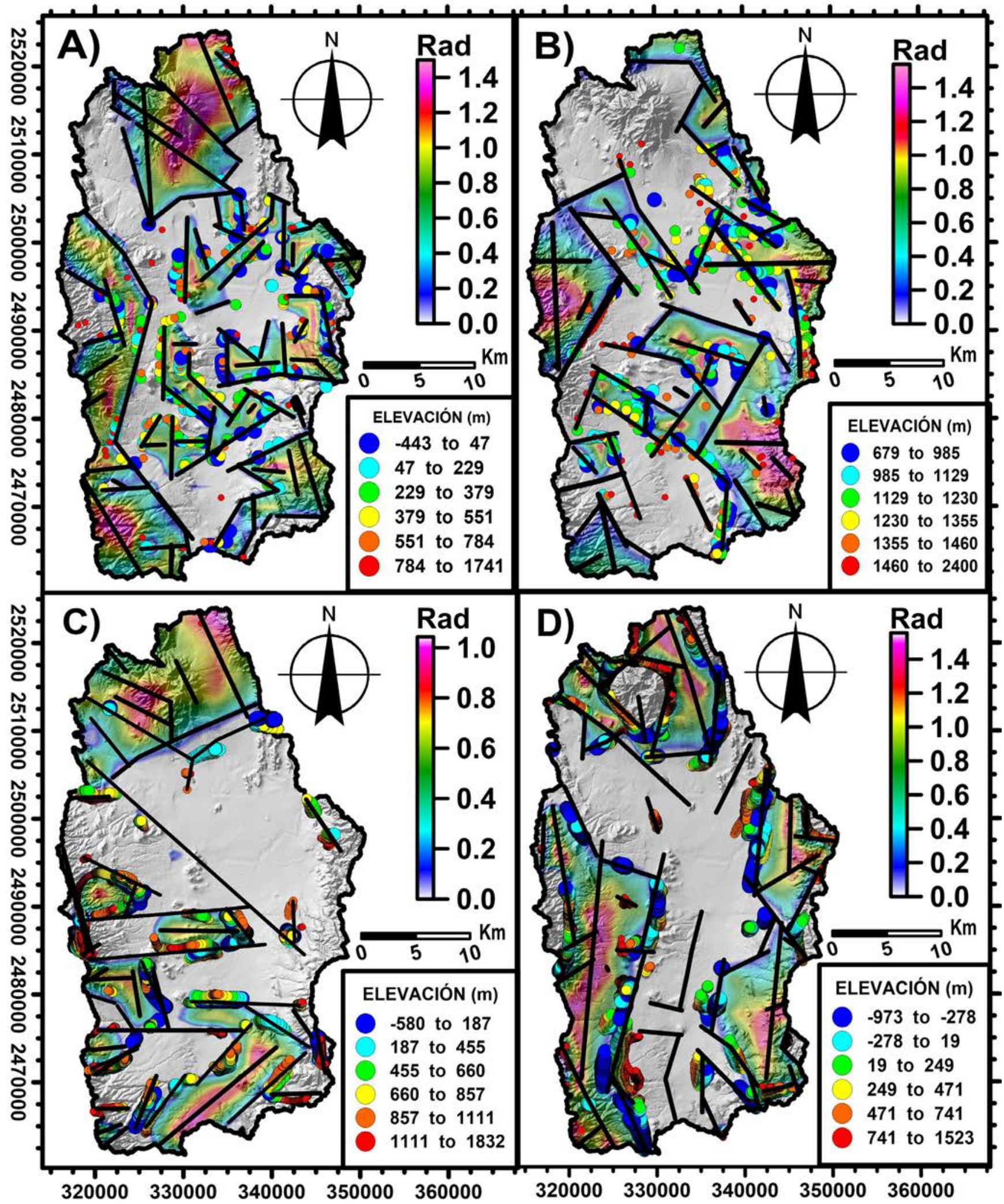

Figura 6 Mapa que muestra en conjunto los resultados de la Deconvolución de Euler (DE), el Angulo de Inclinación (AI) y la Topografía. A) Configuración para la magnetometría terrestre, B) Configuración para la gravimetría terrestre, C) Configuración para la magnetometría aérea, D) Configuración para la gravimetría Satelital. 
ca) compuestas por rocas del Cretácico (Aptiano-Maastrichtiano) y cuyos núcleos presentan una dirección preferencial NW-SE (Figura 5b).

La zona centro-sur, de color cian se asocia a posibles pilares tectónicos a diferentes profundidades, hacia la parte oriental de la CVH se observan los valores más bajos de elevación tanto en el DEM (Figura 5A) como en la magnetometría terrestre (Figura 5C) indicando el cruce en esta zona de los sistemas de fallas NW-SE y N-S (Figuras 5a, 5b y $5 c)$.

Para la configuración de la magnetometría terrestre (Figura 5C) se identificaron elevaciones que varían de los 371 a 1630 msnm Las principales orientaciones de los lineamientos identificados en el mapa son: NW-SE y N-S (Figura 5c) asociándose a fallas del mapa superficial y el mapa de gravimetría terrestre (Figuras 5A y 5B) las cuales han originado diferentes estructuras geológicas en el área de estudio.

En la configuración de la magnetometría aérea (Figura 5D) los valores de elevación del mapa oscilan en un rango entre -360 a 1290 msnm, infiriéndose que los sistemas de lineamientos NW-SE y NE-SW (Figura 5d) son los de mayor orden, así como los más antiguos, el sistema N-S el de menor orden y el más reciente para esta configuración.

Los lineamientos antes mencionados coinciden con regímenes de falla reconocidos en superficie (Figura 5A) y con estructuras de carácter regional como: el cinturón plegado de la Sierra Madre Oriental, el Graben de Arista, Graben de Peotillos, Graben de San Luis, Graben de Villa de Reyes, entre otros. (Labarthe-Hernández, 1982; Barboza-Gudiño et al., 2002; Alaniz-Álvarez y Nieto-Samaniego, 2005; López-Doncel et al., 2007; Tristán-González et al., 2009). Lo anterior indica que la evolución tectónica de la $\mathrm{CVH}$ se ha dado en una serie de etapas/pulsos y que el relieve está asociado a una secuencia sedimentaria y volcánica (Triásica-Jurásica).

En la configuración de la gravimetría satelital (Figura $5 \mathrm{E}$ ) se tienen valores de relieve que varían de los -600 a 300 msnm, los cuales corresponden al horizonte más antiguo de la zona de estudio posiblemente asociado al basamento metamórfico del Precámbrico, teniendo una dirección preferencial de sus lineamientos NW-SE (Figura 5e).

\subsection{LINEAMIENTOS GEOFÍSICOS}

Para la caracterización de los lineamientos se realizaron diversos juegos de mapas en los que se combinaron con la topografia, el algoritmo de AI y se superpusieron los resultados de la DE (Figura 6), permitiendo interpretar diferentes tendencias de dichos lineamientos en los diferentes conjuntos de datos.

En el mapa correspondiente a los datos de la magnetometría terrestre (Figura 6A) la tendencia general de los lineamientos tiene un rumbo principal N-S, seguido de otra tendencia NW-SE y en menor medida un sistema NE-SW que en algunos casos llegan hasta E-W.

Los resultados de la DE indican una buena correlación en la parte central del mapa con los valores del AI iguales a cero debido a que los dos algoritmos identifican la geometría de la fuente. Por lo tanto la DE confirma los resultados de la AI, bajo el supuesto de que los bordes de las fuentes anómalas son causadas por contactos verticales. Esta correlación es una manera de confirmar la ubicación horizontal y la profundidad de bordes que reflejan diferentes atributos de características lineales, tales como fallas, contactos, bordes de cuencas y levantamientos (Oruç, 2011).

La configuración de la gravimetría terrestre (Figura 6B) muestra que el sistema dominante es el NW-SE, así como el N-S y en menor medida el NE-SW, evidenciando que en la parte central del mapa, y en la porción oriental el valor de cero del AI junto con la Deconvolución de Euler tiene una coincidencia adecuada, asociadas a posibles fallas. El mapa de la magnetometría aérea (Figura 6C) tiene pocos lineamientos, dominan los de rumbo E-W y en menor proporción los de rumbo NE- 
SW. En general, el mapa muestra un menor número de lineamientos debido a la detección de anomalías en la zona asociadas a derrames de basaltos, andesitas y posibles intrusivos presentes al Oeste, Norte, NW y NNW de la CVH.

La configuración del mapa de gravimetría satelital (Figura 6D) muestra una tendencia dominante N-S seguida por un sistema NW-SE. Se puede observar que tanto el valor de cero del AI como la Deconvolución de Euler coinciden muy bien en los bordes de la CVH, delimitando de manera general a la Sierras del Coro hacia el Oeste, Álvarez en el SSW, Peotillos al Este y El Peyote al igual que la de La Tapona al norte, indicando lineamientos de carácter regional.

\subsection{INTERPRETACIÓN Y TENDENGIA DE LOS RASGOS LINEALES}

Para poder analizar e interpretar los lineamientos geofísicos-estructurales de manera conjunta, se sumaron los resultados de los diferentes mapas de magnetometría, gravimetría, así como la topografía de la zona de estudio (Figura 7A), posteriormente se hizo el cálculo de la densidad de lineamientos permitiendo interpretar los principales sistemas de fallas (Figura 7B), apoyando una mejor comprensión del marco estructural de la CVH.

En total se analizaron 323 lineamientos identificados, mostrando en el diagrama unidireccional de rosetas de la CVH (Figura 7C) que las direcciones preferenciales de las estructuras son: NW-SE, N-S y NE-SW, siendo las de orientación NW-SE las más dispersas, las de mayor frecuencia y con los lineamientos de mayor longitud.

De acuerdo con la tendencia encontrada se interpreta que el sistema de dirección NW-SE es el más antiguo, ya que afecta a rocas cretácicas tanto de cuenca, que forman parte de la MC, como de plataforma que conforman parte de la SMO, correlacionándose al evento Laramídico que afectó al centro y noreste de México (López-Doncel et al., 2007), el cual posiblemente tuvo una reactivación durante el Oligoceno facilitando las emisiones fisurales de rocas volcánicas, dando a entender que fueron tectónicamente controladas (Tristán-González et al., 2008).

Los lineamientos de rumbo NE-SW se relacionan a sistemas contemporáneos a los últimos esfuerzos de la Orogenia Laramide o posteriores, afectando a las rocas sedimentarias del Cretácico y a la secuencia de rocas volcánicas presentes en el área de estudio.

La tendencia N-S se asocia a rasgos estructurales más recientes relacionados a la formación de fosas y pilares tectónicos (grabens y horsts) continuando hacia el norte de México y Estados Unidos pertenecientes al dominio tectónico de la provincia extensional de Cuencas y Sierras (Basin and Range) (Barboza-Gudiño et al., 2002; López-Doncel et al., 2007, Tristán-González et al., 2008).

A partir de la figura 7A se pudieron interpretar los lineamientos principales (Figura 7B) asociados a diversas estructuras geológicas, apreciándose claramente en el mapa la presencia de una falla de carácter regional la cual se extiende en los bordes de la Sierra de Álvarez hacia el sur y de la Sierra del Coro en el centro y NNW del límite Occidental de la CVH con una longitud que se aproxima a los $41 \mathrm{~km}$, por otro lado se puede observar hacia el límite oriente la presencia de un graben de orientación N-S en su parte norte, cambiando ligeramente al sur a una dirección NW-SE, el cual ha sido denominado Graben de Peotillos, con un ancho de $6 \mathrm{~km}$ en la parte norte y estrechándose a $2 \mathrm{~km}$ en su parte sur, teniendo una longitud aproximada de $27 \mathrm{~km}$. También se identificó la existencia del Graben de Corcovada de rumbo NW-SE con un ancho de $3.6 \mathrm{~km}$ por $12 \mathrm{~km}$ de largo, el cual se cruza con la fosa tectónica de Peotillos en el Valle de Concordia.

Hacia la parte NNW del mapa se tiene una franja de dirección NW-SE, delimitada por lineamientos que pueden estar asociados a fallas, hacia su porción SE se interpone con una depresión, la cual probablemente se desarrolló por el cruce de dicha franja con el sistema de fallas asociado al Graben de Peotillos. Además se tienen lineamientos inferidos en la parte norte y NNE de la cuenca con rumbo NW-SE. 


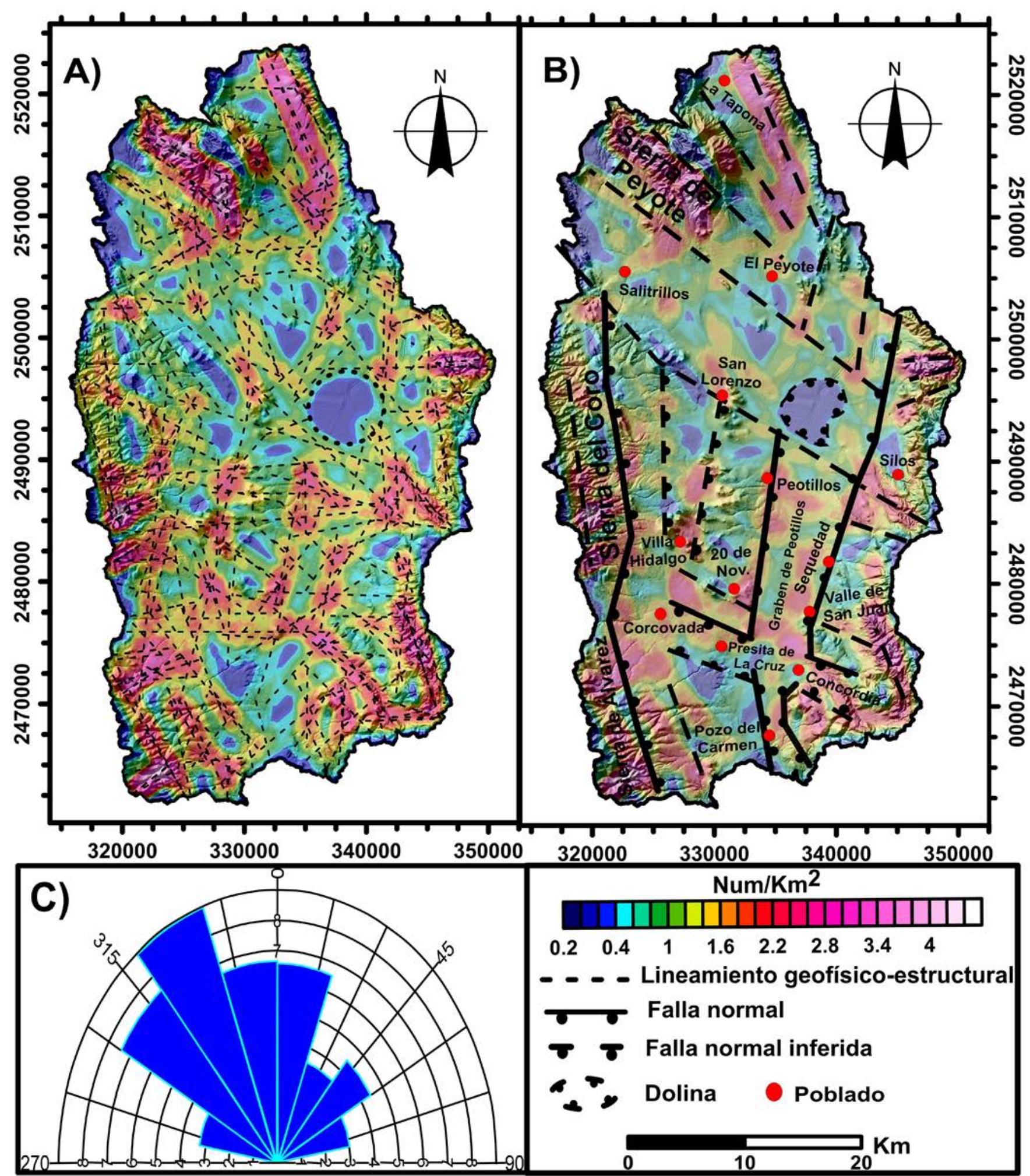

Figura 7 Mapas de Densidad de Lineamientos. A) Plano que muestra el total de las direcciones obtenidas en los diferentes mapas de los métodos potenciales, con el que se calculó la densidad de acuerdo al número de lineamientos por kilómetro cuadrado; B) Plano de lineamientos geofísicos-estructurales principales; C) Diagrama de roseta que muestra que las principales tendencias de los lineamientos son NW-SE. 
El origen de los lineamientos NW-SE está ligado a la Orogenia Laramide y al desarrollo posterior de una zona de cizalla de orientación NNE desarrollada en el límite entre los elementos paleogeográficos de la PVSLP y La CMCM a lo largo del lineamiento Matehuala-San Luis Potosí (Tristán-González et al., 2009) y se localiza al poniente de la zona de estudio.

El movimiento lateral derecho de estos dos bloques provocó una serie de estructuras en échelon y levantamientos de pequeños bloques exponiendo el basamento Triásico en zonas aledañas a la CVH, también causó el desarrollo de fallas con rumbo NW-SE (Tristán-González et al., 2009), las cuales se asocian a los lineamientos con la misma orientación detectados en la zona de estudio los cuales sirvieron como conductos para rocas intrusivas y volcánicas, pudiendo así interpretar posibles fallas antes no consideradas.

Posteriormente la región fue afectada por un periodo de subsidencia formando grábenes de orientación NW-SE ocasionando que las rocas volcánicas y depósitos clásticos continentales quedaran apilados y posteriormente sepultados (Barboza-Gudiño et al., 2002; Tristán-González et al., 2009), sugiriendo la posible interpretación de la Cuenca de Villa Hidalgo como de tipo transtensional.

\subsection{MODELO GEOFÍSICO-GEOLÓGICO (SEGCIÓN A-A')}

Para poder definir la geometría y profundidad de las diferentes estructuras asociadas a las formaciones geológicas de la CVH se realizó el modelado 2D de la Sección A-A' (Figura 2), cuya dirección es NNE-SSW viendo hacia el oeste, situándose a lo largo del graben de Peotillos. Esta zona geológica es la más representativa para estudiar el control estructural del funcionamiento hidrogeológico de la zona.

La modelación se realizó con el software GM-SYS basado en el algoritmo de inversión de Marquardt (Marquardt, 1963), que utiliza diversos cuerpos poligonales de diferente densidad y susceptibilidad magnética (Figura 7). Los valores utilizados para dicha modelación fueron: el CMR reducido al polo de la magnetometría terrestre y aérea, la anomalía residual de Bouguer de la gravimetría terrestre y satelital.

La sección A-A' de acuerdo a las propiedades físicas y morfología de las anomalías (figuras 8A8D) de los diferentes conjuntos de datos, muestra la presencia de 6 diferentes Unidades Geofísicas (UG) principales (Figura 8E).

La primera unidad geofisica (UGl) se encuentra cercana a la superficie a lo largo de toda la sección, presentando valores de densidad (p) de $1.8 \mathrm{~g} / \mathrm{cm}^{3}$ y susceptibilidad magnética $(\boldsymbol{k})$ de $0.2 \times 10^{-3} \mathrm{SI}$. Sus espesores varían de 200 a $300 \mathrm{~m}$ hacia el SSW adelgazándose en la parte central $(100-150$ m), teniendo el mayor espesor hacia el NE que varía de 350 a 400 m, volviéndose a disminuir hacia el extremo NE. La UG1 se asocia a depósitos sedimentarios que van desde limos, arenas finas hasta llegar al tamaño de gravas.

La unidad geofísica (UG2) tiene un valor de $\boldsymbol{\rho}=2.3$ $\mathrm{g} / \mathrm{cm}^{3}$ y $\boldsymbol{k}=0.94 \times 10^{-3} \mathrm{SI}$, abarcando desde el extremo SW de sección hasta acotarse lateralmente hacia el NE en la coordenada UTM-Y $=2489031$. Su espesor promedio es de $150 \mathrm{~m}$ y corresponde a rocas volcánicas de composición riolítica.

La unidad geofísica 3 (UG3) subyace a la anterior y se extiende paralelamente a la UG2, tiene valores de $\boldsymbol{\rho}=2.12 \mathrm{~g} / \mathrm{cm}^{3}$ y una $\boldsymbol{k}=0.2 \times 10^{-3} \mathrm{SI}$, interpretándose como una secuencia sedimentaria del Cretácico superior con un espesor que varía de 200 a $300 \mathrm{~m}$.

La unidad geofísica (UG4) subyace a la UG3 hasta la coordenada UTM-Y 2489031, donde una depresión corta verticalmente a la UG2 y UG3.

La UG4 se extiende a lo largo de toda la sección, teniendo espesores que varían de los $400 \mathrm{~m}$ a los $650 \mathrm{~m}$ siendo la parte NE la de mayor rango, $\boldsymbol{\rho}=$ $2.7 \mathrm{~g} / \mathrm{cm}^{3}$ y el valor de $\boldsymbol{k}=-0.01 \times 10^{-3} \mathrm{SI}$, asociándose a rocas masivas de composición calcárea del Cretácico medio (Albiano-Cenomaniano).

La unidad geofísica 5 (UG5) se extiende a lo largo de toda la sección, subyaciendo a la UG4 con valores de $\boldsymbol{\rho}=2.8 \mathrm{~g} / \mathrm{cm}^{3}, \boldsymbol{k}=0.63 \times 10^{-3}$ SI y un espesor de 400 a $700 \mathrm{~m}$, teniendo el mayor valor al 


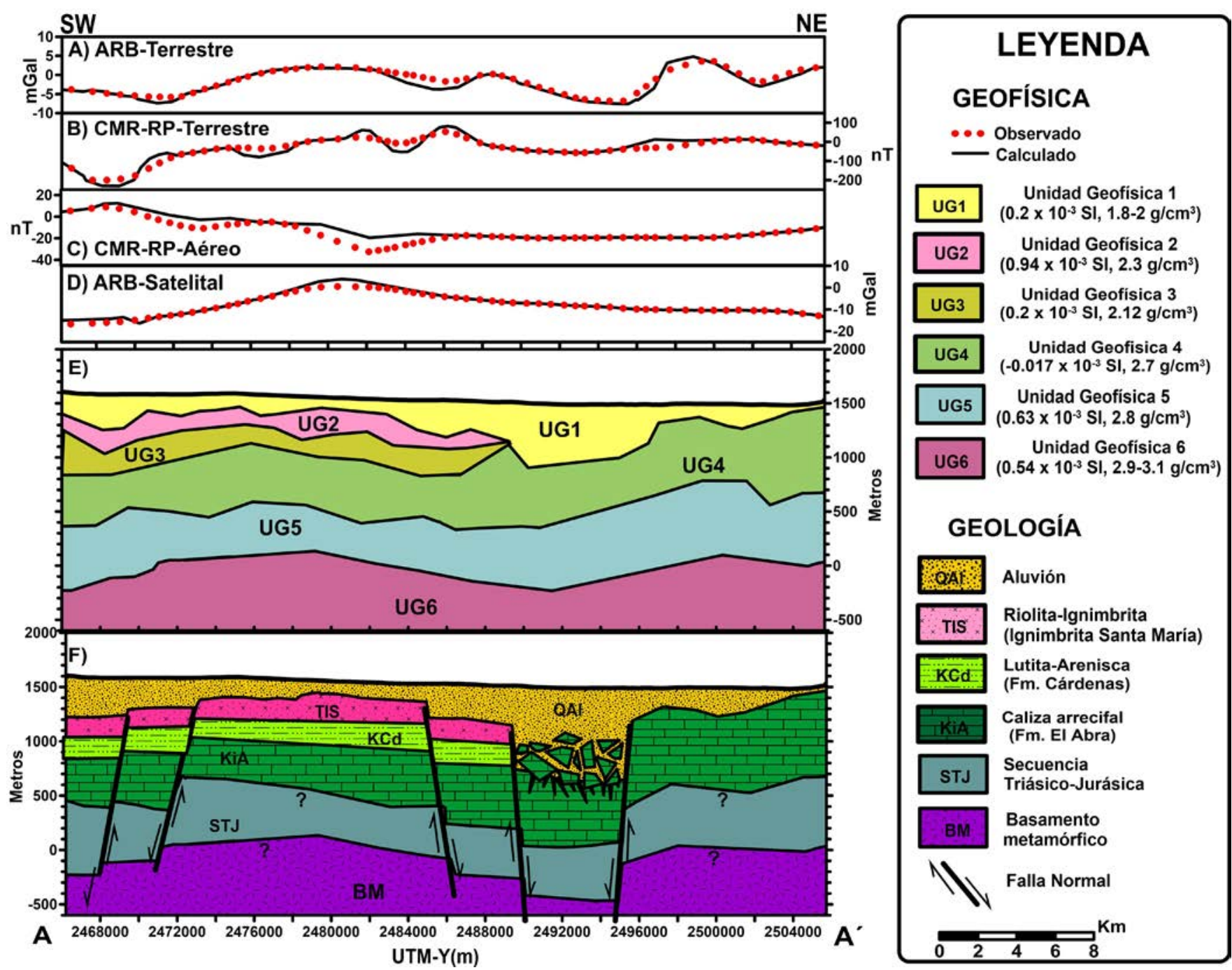

Figura 8 Configuración de la sección A-A', conformando A) Campo observado y calculado del mapa de la Anomalía Residual de Bouguer (ARB) Terrestre; B) Campo observado y calculado del mapa del Campo Magnético Residual Reducido al Polo (CMR-RP) Terrestre; C) Campo observado y calculado del mapa del CMR-RP Aéreo D) Campo observado y calculado del mapa dela ARB Satelital. E) Interpretación de los de unidades geofísicas que constituyen valores de densidad y susceptibilidad magnética. F) Interpretación de la sección geológica del graben de Peotillos.

NE. La UG5 corresponde probablemente a una secuencia Jurásica-Triásica.

La unidad geofísica 6 (UG6) subyace a todas las UG de la sección, teniendo valores de $\boldsymbol{\rho}=3 \mathrm{~g} / \mathrm{cm}^{3}$ y $\boldsymbol{k}$ promedio de $0.54 \times 10^{-3} \mathrm{SI}$.

La UG6 se extiende a lo largo de toda la sección y muestra un levantamiento cerca de la parte centro-SW junto con todas las UG y se interpreta como el posible basamento metamórfico de la zona de estudio.
Se interpretaron 2 conjuntos de fallas normales principales (N-S y NW-SE), asociados a fosas y pilares tectónicos a diferentes profundidades y cubiertos por material aluvial.

Las fallas normales escalonadas corresponden al sistema NW-SE, teniendo un pilar tectónico o levantamiento delimitado por las coordenadas UTM-Y= 2471172 al SW y 268646 al NE (Figura $8 \mathrm{~F})$, dando como resultado que el espesor del relleno sedimentario sea menor en esta zona, variando 
de los $80 \mathrm{~m}$ a los $100 \mathrm{~m}$.

Existe una serie de escalones estructurales en ambos flancos del pilar ocasionando que los espesores del relleno sedimentario aumenten de 200 a $300 \mathrm{~m}$ hacia el SW y de 300 a $400 \mathrm{~m}$ hacia el NE (Figura 8F). Se tiene la presencia de una depresión delimitada por las coordenadas UTM-Y= 2489662 y 2495498 , la cual probablemente vio favorecida su formación por el cruce del graben de Peotillos con otra estructura NW-SE evidenciada en la Figura 7B.

Las secuencias interpretadas como Jurásica-Triásica y el basamento metamórfico no afloran en el área de estudio, únicamente en zonas aledañas (Sierra de Charcas, Sierra de la Ballena, Real de Catorce, entre otras).

\subsection{FUNGIONAMIENTO HIDROGEOLÓGICO}

El agua subterránea se localiza principalmente en tres unidades hidrogeológicas, que a su vez forman diferentes tipos de acuíferos. La primera unidad se localiza en el material de relleno de las fosas constituida por sedimentos lacustres intercalados con conglomerados semiconsolidados de espesores que varían de $100 \mathrm{~m}$ a $300 \mathrm{~m}$, siendo de tipo libre a semiconfinado, y cubiertos por una capa arcillosa que funciona en todo el valle como acuitardo. La segunda unidad corresponde al lecho rocoso constituido por rocas volcánicas de composición riolítica y dacítica fracturadas del Oligoceno y algunos basaltos del Cuaternario clasificándose como un acuífero semiconfinado.

La tercera unidad la conforman calizas arrecifales de la Formación El Abra (Albiano-Cenomaniano), clasificándose como un acuífero cárstico confinado por lutitas y areniscas de la Formación Cárdenas (Maastrichtiano-Campaniano), teniendo un alto potencial hidrogeológico a profundidades que van de los 400 a $600 \mathrm{~m}$ en el graben de Peotillos. Con los valores piezométricos (obtenidos de restar al valor de elevación del brocal de los pozos la profundidad del nivel estático medida en campo), se configuró una malla de valores isopiezométricos con el que se construyó la red de flujo del agua subterránea, mostrando las zonas de recarga localizadas en casi toda la periferia del graben de Peotillos y dirección preferencial S-N (Figura 9A). El agua subterránea se infiltra e interconecta en los diferentes tipos de acuíferos a través de fallas y fracturas del subsuelo, originadas tanto por movimientos extensivos como laterales ocasionando permeabilidad secundaria tanto en las rocas volcánicas como en las rocas sedimentarias del Cretácico presentes en el subsuelo (Figura 9B).

La principal zona de descarga coincide con los valores más bajos de elevación y densidad de la CVH donde en profundidad se vincula con una depresión probablemente conectada a la falla "Montaña" nombrada así por su cercanía al poblado del mismo nombre, estableciendo una interconexión entre el sistema acuífero de Villa Hidalgo y el sistema acuífero de Cerritos.

\section{Conclusiones}

La integración de diferentes datos potenciales junto con la aplicación de la metodología Deconvolución de Euler, el algoritmo matemático del ángulo de Inclinación y el espectro de potencias, permitieron caracterizar de manera eficiente los lineamientos geofísicos-estructurales, así como la identificación del posible basamento metamórfico en la Cuenca de Villa Hidalgo.

El área de estudio se encuentra afectada por diversos sistemas de fallas regionales los cuales de acuerdo a la interpretación del mapa de densidad de lineamientos, se pudieron identificar fallas y estructuras con direcciones preferenciales NW-SE y N-S y no habían sido reportadas.

El origen de los sistemas NW-SE debido a la Orogenia Laramide y a una zona de cizalla desarrollada en el margen oeste de la zona de estudio y los sistemas de dirección N-S están asociados al evento extensional del Basin and Range, sugiriendo la posible interpretación de la Cuenca de Villa Hidalgo como una zona transtensional.

En la sección A-A' ubicada dentro del graben de Peotillos se identificaron fallas escalonadas, un 


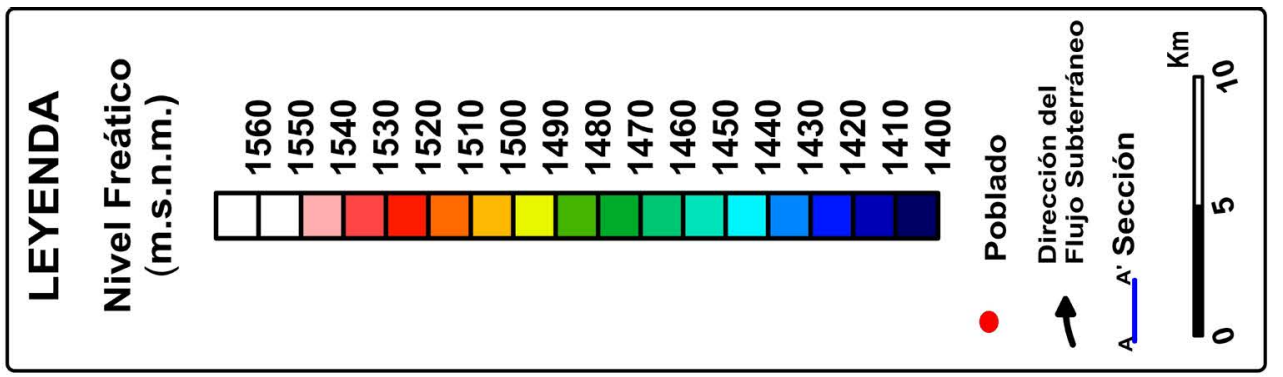

25050002500000249500024900002485000248000024750002470000

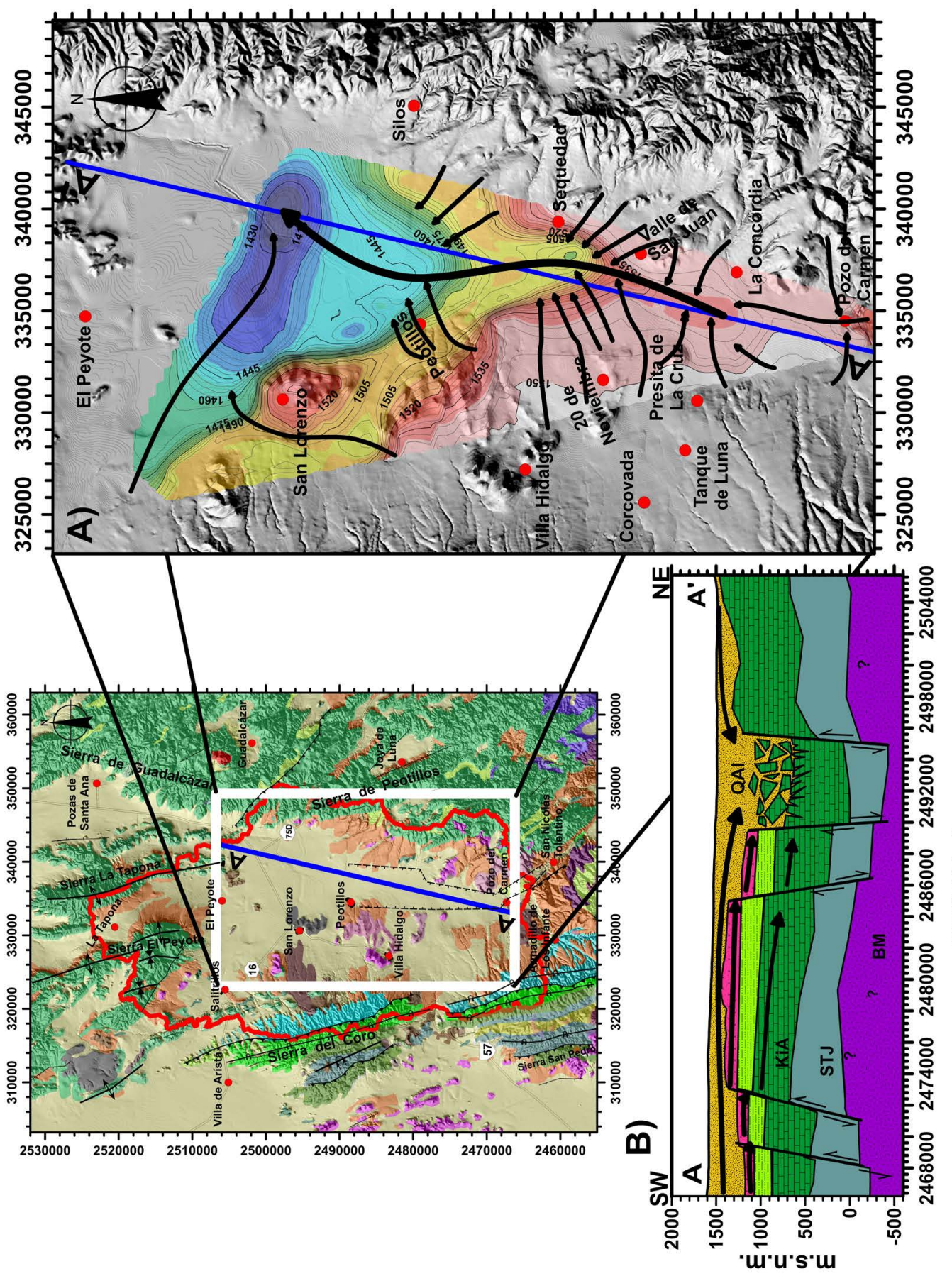

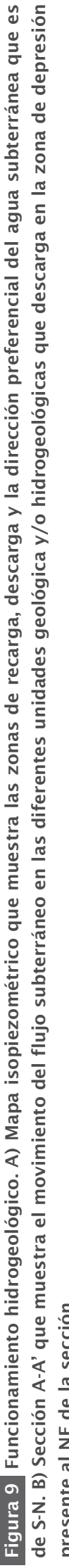


pilar tectónico en el centro y la presencia de una depresión en el NE, los cuales controlan la dinámica del flujo subterráneo en la zona. También se identificaron 2 capas a profundidad, la primera se correlaciona posiblemente a una secuencia Jurásica-Triásica a profundidades que varían de $900 \mathrm{a}$ 1400 metros y la segunda se asocia al basamento metamórfico de la zona de estudio a profundidades de 1600 a 2000 metros.

La piezometría y el modelado geofísico-geológico permitieron reconocer qué bordes del área de estudio actúan como zonas de recarga y el flujo del agua subterránea tiene una dirección preferencial S-N, circulando por las unidades geológicas conformadas en los diferentes tipos de acuíferos a través de las fallas originadas tanto por movimientos extensivos como laterales, fracturando y causando permeabilidad secundaria en las rocas volcánicas y rocas sedimentarias del Cretácico presentes en el subsuelo.

La zona NE es la principal zona de descarga del área debido a los valores más bajos de elevación y densidad de la Cuenca de Villa Hidalgo y se asocia en profundidad con la ubicación de una depresión probablemente conectada a la falla "Montaña", estableciendo una interconexión entre el sistema acuífero de Villa Hidalgo y el Sistema acuífero de Cerritos.

\section{Agradecimientos}

Se agradece al IPICYT por el apoyo brindado en la realización de éste estudio. F. Y. Pérez agradece al CONACYT por la beca de Doctorado otorgada (Registro: 221813). Los autores agradecen también al Dr. Sánchez y otros revisores anónimos por sus comentarios constructivos que permitieron mejorar de manera notable el escrito final.

\section{Referencias}

Alaniz-Álvarez, S.A., Nieto-Samaniego, A.F., 2005, El sistema de fallas Taxco-San Miguel de
Allende y la Faja Volcánica Transmexicana, dos fronteras tectónicas del centro de México activas durante el Cenozoico: Boletín de la Sociedad Geológica Mexicana: LVII (1), 65-82.

Aranda-Gómez, J.J., Labarthe-Hernández, G., 1977, Estudio geológico de la Hoja Villa Hidalgo, S.L.P.: Universidad Autónoma de San Luis Potosí, Instituto de Geología y Metalurgia, Folleto Técnico, 53.

Aranda-Gómez, J.J., Henry, G.D., Luhr, J.F., 2000, Evolución tectono-magmática postpaleocénica de la Sierra Madre Occidental y de la porción meridional de la provincia tectónica de Cuencas y Sierras, México. Boletín de la Sociedad Geológica Mexicana, 53, 59-71.

Baranov, V., Naudy, H., 1964, Numerical calculation of the formula of reduction to the magnetic pole: Geophysics, 29, 67-79.

Barboza-Gudiño, J.R., Zavala-Monsiváis, A., Venegas-Rodriguez, G., Barajas-Nigoche, L.D., 2010, Late Triassic stratigraphy and facies from northeastern Mexico: Tectonic setting and provenance, Geosphere, 6(5), 621-640.

Barboza-Gudiño, J.R., Orozco-Esquivel, M.T., Gómez-Anguiano, M., Zavala-Monsiváis, A., 2008, The Early Mesozoic volcanic arc of western North America in northeastern Mexico: Journal of South American Earth Sciences, 25, 49-63.

Barboza-Gudiño, R., López-Doncel, R., MataSegura, J.L., 2002, Carta Geológico-Minera Villa Hidalgo F14-A74, escala 1:50000: México, Estado de San Luis Potosí, Secretaría de Economía, Consejo de Recursos Minerales y Universidad Autónoma de San Luis Potosí, Instituto de Geología, 42

Beiki, M., Bastani, M., Pedersen, L.B., 2010, Leveling HEM and aeromagnetic data using differential polynomial fitting: Geophysics, 75(1), 13-23.

Bhattacharyya, B.K., 1966, Continuous spectrum of the total-magnetic-field anomaly due to 
a rectangular prismatic body: Geophysics, 31(1), 97-121.

Blakely, R.J., 1995, Potential theory in gravity and magnetic applications: Cambridge, ReinoUnido, Cambridge University Press, $441 \mathrm{p}$.

Bournas, N., Galdeano, A., Hamoudi, M., Baker, H., 2003, Interpretation of the aeromagnetic map of Eastern Hoggar (Algeria) using the Euler deconvolution, analytic signal and local wavenumber methods: Journal of African Earth Sciences, 37, 191-205.

Burger, H.R., 1992, Exploration geophysics of the shallow subsurface: New Jersey, E.U.A, Prentice Hall, 489.

Centeno-García, E., 2005, Review of upper Paleozoic and Mesozoic stratigraphy and depositional environments of central and west Mexico: Constraints on terrane analysis and paleogeography, en Anderson, T.H., Nourse, J.A., McKee, J.W., Steiner, M.B. (eds.), The Mojave-Sonora megashear hypothesis: Development, assessment and alternatives: Geological Society of America Special Paper, 393, 233-258.

Chennouf, T., Khattach, D., Milhi, A., Andrieux, P., Keating, P., 2007, Major structural trends in northeastern Morocco: the contribution of gravimetry: Comptes Rendus Geoscience, 339, 383-395.

Coons, R.L., Woollard, G.P., Hershey, G., 1967, Structural Significance and Analysis of Mid-Continent Gravity High: American Association of Petroleum Geologists Bulletin, 51, 2381-2399.

Cordell, L., Grauch, V.J.S., 1985, Mapping basement magnetization zones from aeromagnetic data in the San Juan Basin: New México. Society of Exploration Geophysics, 181-197.

Córdoba-Méndez, D.A., 1964, Geology of Apizolaya quadrangle (east half), northern Zacatecas, Mexico, Tesis de maestria: Austin, University of Texas, $111 \mathrm{p}$.
FitzGerald, D., Reid, A., McInerney, P., 2004, New discrimination techniques for Euler deconvolution. Computers \& Geosciences, 30, 461-469.

Fullea, J., Fernàndez, M., Zeyen, H., 2008, FA2BOUG - A FORTRAN 90 code to compute Bouguer gravity anomalies from gridded free-air anomalies: Application to the Atlantic-Mediterranean transition zone: Computers \& Geosciences, 34(12), 1665-1681.

Hinze, W.J., Von Frese, R.R.B., Saad, A.H., 2013, Gravity and Magnetic Exploration: Principles, Practices, and Applications. Cambridge, U. K., Cambridge University Press, 512.

Hoppe, M., Barboza-Gudiño, J.R., Schulz, H.M., 2002, Late Triassic submarine fan in northwestern San Luis Potosí, MéxicoLithology, facies and diagenesis: Neues Jahrbuch für Geologie und Paläontologie, 2002, 705-724.

Imlay, R.W., Cepeda, E., Álvarez, M., Jr., Diaz, T., 1948, Stratigraphic relations of certain Jurassic formations in eastern Mexico: American Association of Petroleum Geologists Bulletin, 32, 1750-1761.

Labarthe-Hernández, G., Tristán-González, M., Aranda-Gómez, J.J., 1982, Revisión Estratigráfica del Cenozoico de la Parte Central del Estado de San Luis Potosí: Universidad Autónoma de San Luis Potosí, Instituto de Geología y Metalurgia, Folleto Técnico, 85, 205.

López-Doncel, R., Soto-Araiza, R.G., DircioCastro, D., 2007, Carta Geológico-Minera Peotillos F14 A75, escala 1:50000, Estado de San Luis Potosí: Secretaria de Economía, Servicio Geológico Mexicano y Universidad Autónoma de San Luis Potosí, Instituto de Geología, 67.

Lu, R.S., Mariano, J., Willen, D.E., 2003, Differential reduction of magnetic anomalies to the pole on a massively parallel computer: Geophysics, 68(6), 1945-1951. 
Marquardt, D.W., 1963, An algorithm for leastsquares estimation of nonlinear parameters: Journal of the Society for Industrial and Applied Mathematics, 11, 431-441.

Miller, H.G., Singh, V., 1994, Potential field tilt-a new concept for location of potential field sources: Journal of Applied Geophysics, 32, 213-217.

Mishra, D.C., Naidu, P.S., 1974, Two-dimensional power spectral analysis of aeromagnetic fields: Geophysical Prospecting, 22, 345-353.

Mixon, R.B., Murray, G.E., Diaz, T.G., 1959, Age and correlation of Huizachal Group (Mesozoic), state of Tamaulipas, Mexico: American Association of Petroleum Geologists Bulletin, 43, 757-771.

Nieto-Samaniego, A.F., Alaniz-Álvarez, S.A., Camprubí, A., 2005, La Mesa Central de México: estratigrafía, estructura y evolución tectónica cenozoica: Boletín de la Sociedad Geológica Mexicana, 57, 285-318.

NOAA. 2010. IGRF. Recuperado de http://www. ngdc.noaa.gov/IAGA/vmod/igrf.html.

Oruç, B., 2011, Edge Detection and Depth Estimation Using a Tilt Angle Map from Gravity Gradient Data of the Kozali-Central Anatolia Region, Turkey: Pure and Applied Geophysics, 168, 1769-1780.

Pavlis, N.K., Holmes, S.A., Kenyon, S.C., Factor, J.K., 2012, The development and evaluation of the Earth Gravitational Model 2008 (EGM2008): Journal of Geophysical Research: Solid Earth, 117, 1-38.

Martínez-Pérez, J., 1972, Exploración geológica del área El Estribo-San Francisco, San Luis Potosí: Boletín de la Asociación Mexicana de Geólogos Petroleros, 24, 327-402.

Ramos-Leal, J.A., López-Loera, H., MartínezRuiz, V.J, Aranda-Gomez, J.J., 2007, Sucesión de eventos y geometría de la parte central del acuífero del graben de Villa de Reyes (San Luis Potosí, México) inferida a partir de datos geoeléctricos: Revista Mexicana de Ciencias Geológicas, 24(1), 31-46.
Reddi, A.G.B., Mathew, M.P., Baldau, S., Naidu, P.S., 1988, Aeromagnetic evidence of crustal structure in the granulite terrane of Tamil Nadu-Kerala: Journal of Geological Society of India, 32, 368-381.

Reid, A.B., Allsop, J.M., Granser, H., Millett, A.J., Somerton, I.W., 1990, Magnetic interpretation in three dimensions using Euler deconvolution: Geophysics, 55, 80-91.

Reid, A.B., FitzGerald, D., McInerney, P., 2003, Euler Deconvolution of gravity data. Society of Exploration Geophysicists (SEG): Annual Meeting, 580-583.

Reynolds, R.L., Rosenbaum, J.G., Hudson, M.R., Fishman, N.S., 1990, Rock magnetism, the distribution of magnetic minerals in the earth's crust, and magnetic anomalies in geological applications of modern aeromagnetic surveys: United States Geological Survey Bulletin, 1924, 24-46.

Schaaf, P., Heinrich, W., Besch, T., 1994, Composition in Sm-Nd isotopic data of the lower crust beneath San Luis Potosí, central Mexico: Evidence from a granulitefacies xenolith suite: Chemical Geology, 118, 63-84.

Shepherd, T., Bamber, J.L., Ferraccioli, F., 2006, Subglacial geology in Coats Land, East Antarctica, revealed by airborne magnetics and radar sounding: Earth Planet Science Letters, 244, 323-335.

Silva-Romo, G., Arellano-Gil, J., MendozaRosales, C., Nieto-Obregón, J., 2000, A submarine fan in the Mesa Central, Mexico: Journal of South American Earth Sciences, 13, 429-442.

Silva-Romo, G., 1994, Estudio de la estratigrafía y estructuras tectónicas de la Sierra de Salinas, estados de San Luis Potosí y Zacatecas, Tesis de Maestría: Facultad de Ciencias, Universidad Nacional Autónoma de México, 144.

Spector, A., Grant, F.S., 1970, Statistical models for interpreting aeromagnetic data: Geophysics, 35(2), 293-302. 
Thompson, D.T., 1982, EULDPH: A new technique for making depth estimates from magnetic data: Geophysics, 47(1), 31-37.

Tristán-González, M., Aguirre-Díaz, G.J., Labarthe-Hernández, G., TorresHernández, J.R., Bellon, H., 2008, PostLaramide and pre-Basin and Range deformation and implications for Paleogene (55-25 Ma) volcanism in central Mexico: A geological basis for a volcano-tectonic stress model: Tectonophysics, 471(1), 136-152.

Tristán-González, M., Aguillón-Robles, A., Barboza-Gudiño, J.R., Torres-Hernández, J.R., Hervé Bellon, H., López-Doncel, R., Rodríguez-Ríos, R., Labarthe-Hernández, G., 2009, Geocronología y distribución espacial del vulcanismo en el Campo Volcánico de San Luis Potosí: Boletín de la Sociedad Geológica Mexicana, 61(3), 287-303.

Verduzco, B., Fairhead, J.D., Green., G.M., MacKenzie, G., 2004, New Insights into Magnetic Derivatives for Structural Mapping: The Leading Edge, 23, 116-119.

Zapata-Zapata, J.L., Pérez-Venzor, J.A., 1979, Cartografía Geológica de la Hoja Peotillos, S.L.P.: Universidad Autónoma de San Luis Potosí, Instituto de Geología y Metalurgia, Folleto Técnico, 63, 30.

Zavala-Monsiváis, A., Barbosa-Gudiño, J.R., Velasco-Tapia, F., García-Arreola, M.E., 2012, Sucesión Volcánica Jurásica en el área de Charcas, San Luis Potosí: Contribución al entendimiento del Arco Nazas en el Noreste de México: Boletín de la Sociedad Geológica Mexicana, 64(3), 277-293. 